The University of Southern Mississippi

The Aquila Digital Community

Faculty Publications

6-1-1998

\title{
The Effect of Convection on a Propagating Front with a Liquid Product: Comparison of Theory and Experiments
}

\author{
B. McCaughey \\ University of Southern Mississippi \\ John A. Pojman \\ University of Southern Mississippi \\ C. Simmons \\ University of Southern Mississippi
}

V.A. Volpert

Université Lyon

Follow this and additional works at: https://aquila.usm.edu/fac_pubs

Part of the Chemistry Commons

\section{Recommended Citation}

McCaughey, B., Pojman, J. A., Simmons, C., Volpert, V. (1998). The Effect of Convection on a Propagating Front with a Liquid Product: Comparison of Theory and Experiments. Chaos, 8(2), 520-529.

Available at: https://aquila.usm.edu/fac_pubs/4995

This Article is brought to you for free and open access by The Aquila Digital Community. It has been accepted for inclusion in Faculty Publications by an authorized administrator of The Aquila Digital Community. For more information, please contact Joshua.Cromwell@usm.edu. 


\title{
The effect of convection on a propagating front with a liquid product: Comparison of theory and experiments
}

\author{
B. McCaughey, J. A. Pojman, and C. Simmons \\ Department of Chemistry and Biochemistry, University of Southern Mississippi, Hattiesburg, \\ Mississippi 39406 \\ V. A. Volpert \\ Analyse numérique, UMR 5585 CNRS, Université Lyon I, 69622 Villeurbanne, France
}

(Received 3 November 1997; accepted for publication 3 February 1998)

This work is devoted to the investigation of propagating polymerization fronts converting a liquid monomer into a liquid polymer. We consider a simplified mathematical model which consists of the heat equation and equation for the depth of conversion for one-step chemical reaction and of the Navier-Stokes equations under the Boussinesq approximation. We fulfill the linear stability analysis of the stationary propagating front and find conditions of convective and thermal instabilities. We show that convection can occur not only for ascending fronts but also for descending fronts. Though in the latter case the exothermic chemical reaction heats the cold monomer from above, the instability appears and can be explained by the interaction of chemical reaction with hydrodynamics. Hydrodynamics changes also conditions of the thermal instability. The front propagating upwards becomes less stable than without convection, the front propagating downwards more stable. The theoretical results are compared with experiments. The experimentally measured stability boundary for polymerization of benzyl acrylate in dimethyl formamide is well approximated by the theoretical stability boundary. (c) 1998 American Institute of Physics. [S1054-1500(98)00502-3]

Exothermic chemical reactions with sufficiently high activation energies can propagate through the medium via a localized reaction zone. The best known example is provided by gaseous flames. In the case of frontal polymerization the localized chemical reaction is polymerization. In many cases propagation of polymerization fronts is accompanied by various physical phenomena and instabilities. In this work we study theoretically and experimentally the influence of natural convection on propagating polymerization fronts. We find conditions when the convective or thermal instabilities appear and compare experimental and theoretical results. They agree surprisingly well.

\section{INTRODUCTION}

Frontal polymerization is a polymerization process where the reaction zone is localized in space. It can propagate through an unmovable medium converting a monomer into a polymer, or the medium itself can move towards the reaction zone as is the case for continuous reactors of frontal polymerization.

Propagating polymerization fronts have many features in common with combustion processes. However, the values of thermophysical parameters, phase states of the reactants and the products of the reaction, and accompanying physical processes such as boiling or melting of the monomer, fingering, crystallization or solidification of the polymer, etc., can essentially distinguish frontal polymerization from other processes connected with propagation of reaction fronts.
Investigation of frontal polymerization begins with the works by Chechilo and Enikolopyan. ${ }^{1-4}$ The authors studied experimentally polymerization of methyl methacrylate and showed that the polymerization process could be fulfilled in the regime of a propagating reaction front. The first experimental works were followed by a large number of experimental and theoretical studies where different polymerizing systems and different models were analyzed. ${ }^{5-7}$

Already in the first works on frontal polymerization it was found that the front propagation can be connected with different forms of instabilities. Now four types of instabilities of polymerization fronts are known: thermal, ${ }^{6-8}$ convective, ${ }^{1,9}$ hydrodynamical, ${ }^{9,10}$ and Rayleigh-Taylor instability (or fingering). 6 ,9

Thermal instability appears because of the competition between the heat production in the reaction zone and the heat diffusion from the reaction zone to the cold reactants. Experimentally it was observed first for the condensed phase combustion $^{11}$ and then studied numerically ${ }^{12-17}$ and analytically. ${ }^{18-21}$ It leads to the appearance of periodic modes of propagation such as one-dimensional pulsations or different temporal, multidimensional structures. For the gaseous combustion thermodiffusional instability can lead also to the appearance of cellular flames. ${ }^{22-25}$ Thermal instability of polymerization fronts was also found and studied in a number of works. ${ }^{6-8}$

Convective instability appears as a result of the action of gravity. If the reaction is exothermic and the reactants are in the liquid phase, then natural convection can occur. For ascending fronts there is some analogy with the RayleighBénard convection in a layer of a liquid heated from below. 
The exothermic chemical reaction plays the role of heating the liquid from below and if the Rayleigh number is sufficiently large, the instability occurs. For the frontal polymerization it is observed experimentally. ${ }^{1,9}$ For the gaseous combustion it is known that the natural convection can influence conditions of the cellular instability ${ }^{26}$ and of the hydrodynamical instability. ${ }^{25}$ There is also a number of works devoted to the interaction of isothermal or weakly exothermal chemical waves with the natural convection. ${ }^{27-36}$ There are some studies of convective instability in chemical reactors. ${ }^{37-39}$

Hydrodynamical instability results from the heat expansion of the medium at the reaction zone. It is well studied for the gaseous combustion. ${ }^{24,25,40} \mathrm{We}$ emphasize that contrary to the convective instability it can appear without gravity, and its physical mechanism is absolutely different. For the frontal polymerization it was observed experimentally ${ }^{9}$ and studied theoretically. ${ }^{10}$

Finally, the Rayleigh-Taylor instability is an instability of the interface between two liquids. There are experimental observations of this instability for frontal polymerization. ${ }^{6,9}$ It can lead to descending particles or drops of the polymer from the reaction zone, i.e., to fingering. In some cases it causes the front degeneration and initiates reaction in the bulk. There are not yet theoretical studies of reactive fingering.

In this work we study the convective instability and its interaction with the thermal instability for propagating polymerization fronts where both the monomer and the polymer are liquid. This paper continues the series of works where we study the stability of polymerization fronts and compare the theory with the experiments. ${ }^{6-8,10,29,41-47}$

The contents of the paper are as follows. In the next section we present the mathematical model and the results of linear stability analysis in a short form sufficient for further comparison with experimental results. The detailed analysis is published elsewhere. ${ }^{43}$ In Sec. III we describe the experiments and compare the experimental results with the theory. We discuss the results in Sec. IV.

\section{MATHEMATICAL MODEL AND LINEAR STABILITY ANALYSIS}

We consider the model that includes equations for the temperature and the concentration in the case of one-step chemical reaction, and the Navier-Stokes equations under the Boussinesq approximation:

$$
\begin{aligned}
& \frac{\partial T}{\partial t}+v \nabla T=\kappa \Delta T+q W, \\
& \frac{\partial \alpha}{\partial t}+v \nabla \alpha=W, \\
& \frac{\partial v}{\partial t}+(v \nabla) v=-\frac{1}{\rho} \nabla p+\nu \Delta v+g \beta\left(T-T_{0}\right) \gamma, \\
& \operatorname{div} \quad v=0 .
\end{aligned}
$$

Here $T$ is the temperature, $\alpha$ is the conversion, $v$ the velocity of the medium, $p$ the pressure, $\kappa$ the coefficient of thermal diffusivity, $q$ the adiabatic temperature change, $\rho$ the density, $\nu$ the viscosity, $g$ the acceleration of gravity, $\beta$ the coefficient of thermal expansion, $T_{0}$ the average value of temperature, $\gamma$ the unit vector in the vertical direction, $\Delta$ $=\partial^{2} / \partial x^{2}+\partial^{2} / \partial z^{2}, \nabla=(\partial / \partial x, \partial / \partial z),-\infty<x, z<\infty . W$ is the reaction rate. Usually it is considered in the form

$$
W=k e^{-E / R_{0} T} \phi(\alpha), \quad \phi(\alpha)=(1-\alpha)^{n},
$$

where $k$ is pre-exponential factor, $E$ is the activation energy, $R_{0}$ the gas constant, and $n$ the order of the reaction.

The boundary conditions are

$z=+\infty: T=T_{i}, \quad \alpha=0, \quad v=0, \quad z=-\infty: \frac{\partial T}{\partial z}=0, \quad v=0$

for the front propagating upwards, and

$z=-\infty: T=T_{i}, \quad \alpha=0, \quad v=0, \quad z=+\infty: \frac{\partial T}{\partial z}=0, \quad v=0$

for the front propagating downwards.

In the equation for the concentration the diffusion term is neglected because the mass diffusion is very slow for polymerization processes. The coupling of Eqs. (2.1) and (2.2) with the equations of motion (2.3) and (2.4) is due to the last term in the right-hand side of (2.3). If the coefficient of thermal expansion $\beta$ is zero and initially the medium is unmovable, then we obtain a simplest model of the condensed phase combustion (2.1) and (2.2).

If $\beta$ is not zero, then the complete system (2.1)-(2.4) should be considered. For the gaseous combustion the Boussinesq approximation was derived and was used to study the influence of gravity on the cellular instability. ${ }^{26,47}$

Using nondimensional variables and parameters

$$
\begin{aligned}
& x_{1}=x \frac{c}{\kappa}, \quad z_{1}=z \frac{c}{\kappa}, \quad t_{1}=t \frac{\kappa}{c^{2}}, \quad v_{1}=\frac{v}{c}, \\
& p_{1}=\frac{p}{c^{2} \rho}, \quad \theta=\frac{T-T_{b}}{q}, \quad \theta_{0}=\frac{T_{b}-T_{0}}{q}, \quad T_{b}=T_{i}+q, \\
& c^{2}=\frac{k \kappa}{q} \frac{R_{0} T_{b}^{2}}{E} e^{-E / R_{0} T_{b}}, \quad P=\frac{\nu}{\kappa}, \quad R=\frac{g \beta q \kappa^{2}}{\nu c^{3}}, \\
& Z=\frac{q E}{R_{0} T_{b}^{2}}, \quad \delta=\frac{R_{0} T_{b}}{E}
\end{aligned}
$$

and omitting the index 1 , we can rewrite the problem (2.1)(2.5) in the form

$$
\begin{aligned}
& \frac{\partial \theta}{\partial t}+v \nabla \theta=\Delta \theta+Z e^{\theta /\left(Z^{-1}+\delta \theta\right)} \phi(\alpha), \\
& \frac{\partial \alpha}{\partial t}+v \nabla \alpha=Z e^{\theta /\left(Z^{-1}+\delta \theta\right)} \phi(\alpha), \\
& \frac{\partial v}{\partial t}+(v \nabla) v=-\nabla p+P \Delta v+P R\left(\theta+\theta_{0}\right) \gamma, \\
& \operatorname{div} \quad v=0,
\end{aligned}
$$


$z=+\infty: \theta=-1, \quad \alpha=0, \quad v=0, \quad z=-\infty: \frac{\partial \theta}{\partial z}=0, \quad v=0$

The boundary condition (2.6) has the form

$z=-\infty: \theta=-1, \quad \alpha=0, \quad v=0, \quad z=+\infty: \frac{\partial \theta}{\partial z}=0, \quad v=0$.

We note that $P$ is the Prandtl number, $Z$ the Zeldovich number, $R$ is a nondimensional parameter which can be called the frontal Rayleigh number. If we introduce the width of the preheat zone $h=\kappa / c$ and the characteristic temperature gradient $A=q / h$, then it takes the form

$$
R=\frac{g \beta A h^{4}}{\kappa \nu}
$$

and coincides with the usual Rayleigh number.

The problems (2.7)-(2.11) and (2.7)-(2.10), (2.12) have a traveling wave solution of the form

$$
\theta(x, z, t)=\tilde{\theta}(z-u t), \quad \alpha(x, z, t)=\tilde{\alpha}(z-u t), \quad v=0 .
$$

Here $u$ is the wave velocity. We study the stability of this traveling wave solution.

We study further in this section stability of reaction fronts with respect to small perturbations. We use an analytical approach based on the infinitely narrow reaction zone method. This method was suggested by Zeldovich and Frank-Kamentsky for gaseous combustion ${ }^{48}$ and later developed for reaction fronts in a condensed medium. ${ }^{49}$ The idea of the method is as follows. If the activation energy $E$ of the chemical reaction is sufficiently large, then the reaction takes place in a narrow reaction zone. Outside of this reaction zone the reaction rate $W$ is small, and this term in Eqs. (2.1) and (2.2) can be neglected. In the limit of an infinitely narrow reaction zone we obtain an interface problem where the reaction term is omitted and replaced by some jump conditions at the interface. This approach can be justified by matching asymptotic expansions. ${ }^{50}$

We reduce the complete model $(2.7)-(2.10)$ to the interface problem. Let the front be located at $z=\zeta(x, t)$ and propagate upwards. Then for $z \neq \zeta(x, t)$

$$
\begin{aligned}
& \frac{\partial \theta}{\partial t}+v \nabla \theta=\Delta \theta, \\
& \alpha=\left\{\begin{array}{lll}
0 & \text { if } & z>\zeta(x, t) \\
1 & \text { if } & z<\zeta(x, t),
\end{array}\right. \\
& \frac{\partial v}{\partial t}+(v \nabla) v=-\nabla p+P \Delta v+P R\left(\theta+\theta_{0}\right) \gamma,
\end{aligned}
$$

$\operatorname{div} v=0$.

The jump conditions at $z=\zeta(x, t)$ have the form:

$$
\begin{aligned}
& {[\theta]=0,\left[\theta^{\prime}\right]=\frac{q}{\kappa} \frac{\partial \zeta}{\partial t}, \quad\left[\theta^{\prime 2}\right]=-2 \frac{q}{\kappa} \int^{\left.\theta\right|_{\zeta}} K(T) d T} \\
& {\left[v_{z}\right]=\left[v_{z}^{\prime}\right]=\left[v_{z}^{\prime \prime}\right]=\left[v_{z}^{\prime \prime \prime}\right]=0 .}
\end{aligned}
$$

Here $[\cdot]$ denotes the jump of a function at the reaction zone, $[f]=f(\zeta-0)-f(\zeta+0)$, and $\theta^{\prime}=\partial \theta / \partial z$.

To complete the problem we should specify also the conditions at infinity:

$z=+\infty: \theta=-1, \quad \alpha=0, \quad v=0 ; \quad z=-\infty: \frac{\partial \theta}{\partial z}=0, \quad u=0$.

For the front propagating downwards the conditions at $+\infty$ and $-\infty$ replace each other.

The derivation of the interface problem (2.13)-(2.19) from (2.7)-(2.11) is given in Ref. 43.

The problem (2.13)-(2.19) has a traveling wave solution

$$
\begin{aligned}
& \theta_{s}\left(z_{1}\right)= \begin{cases}0 & \text { if } \quad z_{1}<0 \\
e^{-c z_{1}-1} \quad \text { if } & z_{1}>0,\end{cases} \\
& \alpha_{s}\left(z_{1}\right)=\left\{\begin{array}{lll}
1 & \text { if } \quad z_{1}<0 \\
0 & \text { if } \quad z_{1}>0,
\end{array}\right. \\
& v_{s}\left(z_{1}\right)=0,
\end{aligned}
$$

where $z_{1}=z-c t$. We note that it is a stationary solution of the problem (2.13)-(2.19) written in the moving coordinates attached to the front. To study the stability of this solution, we linearize the problem about it. We put

$$
\theta=\theta_{s}+\tilde{\theta}, \quad p=p_{s}+\tilde{p}, \quad v=v_{s}+\tilde{v}
$$

and obtain equations for the perturbations $\tilde{\theta}, \tilde{p}, \tilde{v}$ $=\left(\tilde{v}_{x}, \tilde{v}_{z}\right)$ for $z_{1} \neq \xi(x, t)=\zeta(x, t)-c t$ :

$$
\begin{aligned}
& \frac{\partial \widetilde{\theta}}{\partial t}=\Delta \widetilde{\theta}+c \frac{\partial \widetilde{\theta}}{\partial z_{1}}-\tilde{v}_{z} \theta_{s}^{\prime}, \\
& \frac{\partial \tilde{v}}{\partial t}=-\nabla \tilde{p}+P \Delta \tilde{v}+c \frac{\partial \tilde{v}}{\partial z_{1}}+P R \tilde{\theta} \gamma, \\
& \operatorname{div} \quad \tilde{v}=0 .
\end{aligned}
$$

We look for the perturbation in the form

$$
\begin{aligned}
& \tilde{\theta}\left(x, y, z_{1}, t\right)=\left\{\begin{array}{lll}
\tilde{\theta}_{1}\left(z_{1}\right) & \exp (\omega t+i k x), & z_{1}<\xi \\
\tilde{\theta}_{2}\left(z_{1}\right) & \exp (\omega t+i k x), & z_{1}>\xi,
\end{array}\right. \\
& \tilde{v}\left(x, y, z_{1}, t\right)=\left\{\begin{array}{lll}
\tilde{v}_{1}\left(z_{1}\right) & \exp (\omega t+i k x), & z_{1}<\xi \\
\tilde{v}_{2}\left(z_{1}\right) & \exp (\omega t+i k x), & z_{1}>\xi,
\end{array}\right. \\
& \xi(x, y, t)=\epsilon \exp (\omega t+i k x) .
\end{aligned}
$$

Here $\epsilon$ is the amplitude, $\omega$ the frequency, and $k$ the wave number.

We apply the operator rotation two times to Eq. (2.24) and substitute the perturbations in the form given above. We obtain the following equations for the perturbations:

$$
\begin{aligned}
& P \widetilde{v}_{1}^{\prime \prime \prime \prime}+c \widetilde{v}_{1}^{\prime \prime \prime}-\left(2 P k^{2}+\omega\right) \widetilde{v}_{1}^{\prime \prime}-u_{0} k^{2} \widetilde{v}_{1}^{\prime}+k^{2}\left(P k^{2}+\omega\right) \tilde{v}_{1} \\
& \quad=Q k^{2} \widetilde{\theta}_{1}, \\
& \widetilde{\theta}_{1}^{\prime \prime}+c \widetilde{\theta}_{1}^{\prime}-\left(k^{2}+\omega\right) \widetilde{\theta}_{1}=0
\end{aligned}
$$

for $z_{1}<0$ and 


$$
\begin{aligned}
& \begin{array}{l}
P \widetilde{v}_{2}^{\prime \prime \prime}+c \widetilde{v}_{2}^{\prime \prime \prime}-\left(2 P k^{2}+\omega\right) \tilde{v}_{2}^{\prime \prime}-u_{0} k^{2} \widetilde{v}_{2}^{\prime}+k^{2}\left(P k^{2}+\omega\right) \tilde{v}_{2} \\
\quad=Q k^{2} \widetilde{\theta}_{2}
\end{array} \\
& \widetilde{\theta}_{2}^{\prime \prime}+c \widetilde{\theta}_{2}^{\prime}-\left(k^{2}+\omega\right) \widetilde{\theta}_{2}=\widetilde{v}_{2} \theta_{s}^{\prime} \\
& \text { for } z_{1}>0
\end{aligned}
$$

Linearization of the jump conditions $(2.17)$, (2.18) gives

$$
\begin{aligned}
& \widetilde{\theta}_{2}(0)-\widetilde{\theta}_{1}(0)=\epsilon c, \\
& \widetilde{\theta}_{2}^{\prime}(0)-\widetilde{\theta}_{1}^{\prime}(0)=-\epsilon\left(\omega+c^{2}\right)+\widetilde{v}_{1}(0), \\
& c^{2} \epsilon+\widetilde{\theta}_{2}^{\prime}(0)=-\frac{Z}{c} \widetilde{\theta}_{1}(0), \\
& \tilde{v}_{1}(0)=\widetilde{v}_{2}(0), \quad \widetilde{v}_{1}^{\prime}(0)=\widetilde{v}_{2}^{\prime}(0), \quad \widetilde{v}_{1}^{\prime \prime}(0)=\widetilde{v}_{2}^{\prime \prime}(0), \\
& \quad \tilde{v}_{1}^{\prime \prime \prime}(0)=\widetilde{v}_{2}^{\prime \prime}(0) .
\end{aligned}
$$

We require finally that the perturbations decay at infinity:

$$
\tilde{\theta}_{1}(-\infty)=0, \quad \tilde{\theta}_{2}(+\infty)=0, \tilde{v}_{1}(-\infty)=0, \tilde{v}_{2}(+\infty)=0 .
$$

The problem (2.26)-(2.34) has a zero solution. Condition of its nontrivial solvability allows us to find the eigenvalues $\omega$. If all eigenvalues are in the left half plane, then the stationary solution is stable. If there exist eigenvalues in the right half plane, it is unstable. The stability boundary can be found from the condition that the eigenvalue with the maximal real part is on the imaginary axis. There are two different cases. If the eigenvalue on the imaginary axis is pure imaginary, then it corresponds to the oscillatory instability boundary. If it equals zero, then it is cellular instability boundary.

We look for the solution of the problem (2.26)-(2.34) in the form of exponential series. Taking into account that the perturbations are bounded at infinity, we can write the general solution in the form

$$
\begin{aligned}
& \tilde{\theta}_{1}=b_{1}^{-} s_{1}^{-}, \quad \tilde{\theta}_{2}=b_{1}^{+} s_{1}^{+}+b_{2}^{+} s_{2}^{+}+b_{3}^{+} s_{3}^{+}, \\
& \tilde{v}_{1}=b_{1}^{-} w_{1}^{-}+b_{2}^{-} w_{2}^{-}+b_{3}^{-} w_{3}^{-}, \\
& \tilde{v}_{2}=b_{1}^{+} w_{1}^{+}+b_{2}^{+} w_{2}^{+}+b_{3}^{+} w_{3}^{+} .
\end{aligned}
$$

Here $b_{i}^{ \pm}, \quad i=1,2,3$ are arbitrary constants,

$$
\begin{aligned}
& s_{1}^{-}\left(z_{1}\right)=\exp \left(\sigma_{1}^{-} z_{1}\right), \\
& w_{i}^{-}\left(z_{1}\right)=a_{i}^{-} \exp \left(\sigma_{i}^{-} z_{1}\right), \quad i=1,2,3, \\
& \sigma_{1}^{-}=-\frac{u}{2}+\sqrt{\frac{u^{2}}{4}+k^{2}+\omega}, \quad \sigma_{2}^{-}=k>0, \\
& \sigma_{3}^{-}=-\frac{u}{2 P}+\sqrt{\frac{u^{2}}{4 P^{2}}+k^{2}+\frac{\omega}{P}}, \\
& s_{i}^{+}\left(z_{1}\right)=\sum_{j=1}^{\infty} c_{i j} \exp \left(\sigma_{i j}^{+} z_{1}\right), \\
& w_{i}^{+}\left(z_{1}\right)=\sum_{j=1}^{\infty} a_{i j} \exp \left(\sigma_{i j}^{+} z_{1}\right), \quad i=1,2,3,
\end{aligned}
$$

$$
\begin{aligned}
& \sigma_{11}^{+}=-\frac{u}{2}-\sqrt{\frac{u^{2}}{4}+k^{2}+\omega}, \quad \sigma_{21}^{+}=-k>0, \\
& \sigma_{31}^{+}=-\frac{u}{2 P}-\sqrt{\frac{u^{2}}{4 P^{2}}+k^{2}+\frac{\omega}{P}}, \\
& \sigma_{i j}^{+}=\sigma_{i, j-1}^{+}-c, \quad i=1,2,3, j=2,3, \ldots, \\
& a_{21}=a_{31}=1, \quad c_{11}=1, \quad c_{21}=c_{31}=0, \quad a_{2}^{-}=a_{3}^{-}=1 .
\end{aligned}
$$

The coefficients of the series can be found from Eqs. (2.26)(2.29):

$$
\begin{aligned}
& a_{i j}=\frac{P R k^{2} c_{i j}}{\left(\left(\sigma_{i j}^{+}\right)^{2}-k^{2}\right)\left(P\left(\sigma_{i j}^{+}\right)^{2}+c \sigma_{i j}^{+}-\left(P k^{2}+\omega\right)\right)}, \\
& i=1, j=1,2,3, \ldots, \quad i=2,3, j=2,3, \ldots, \\
& c_{i, j+1}=\frac{-c a_{i j}}{\left(\sigma_{i, j+1}^{+}\right)^{2}+c \sigma_{i, j+1}^{+}-\left(k^{2}+\omega\right)}, \\
& a_{1}^{-}=\frac{P=1,2,3, \ldots, 3, \quad j=1, \ldots k^{2}}{\left(\left(\sigma_{1}^{-}\right)^{2}-k^{2}\right)\left(P\left(\sigma_{1}^{-}\right)^{2}+c \sigma_{1}^{-}-\left(P k^{2}+\omega\right)\right)} .
\end{aligned}
$$

We assume that

$$
\left(\left(\sigma_{i j}^{+}\right)^{2}-k^{2}\right)\left(P\left(\sigma_{i j}^{+}\right)^{2}+c \sigma_{i j}^{+}-\left(P k^{2}+\omega\right)\right) \neq 0
$$

for $i=1, j=1,2,3, \ldots, \quad i=2,3, j=2,3, \ldots$, and

$$
\left(\sigma_{i, j+1}^{+}\right)^{2}+c \sigma_{i, j+1}^{+}-\left(k^{2}+\omega\right) \neq 0
$$

for $i=1,2,3, \quad j=1,2,3, \ldots$.

We note that the series defined above converge, and the corresponding solutions are linearly independent if $P \neq 1$ and $P \neq \infty$.

We recall that there are six unknown constants $b_{i}^{ \pm}$and also an unknown amplitude $\epsilon$. They can be found from seven equations (2.30)-(2.33). Substituting solutions (2.35) and (2.36) in (2.30)-(2.33), we obtain a linear algebraic system of equations. To obtain the condition of nontrivial solvability, we set the determinant $\operatorname{det}(R, P, c, k)$ of the system equal to zero. The equation

$$
\operatorname{det}(R, P, c, k)=0
$$

was solved numerically. In the cases $\omega=i \phi$ or $\omega=0$ it gives the oscillatory (Fig. 1) and cellular (Fig. 2) stability boundaries, respectively. In Fig. 1 the critical value of the Zeldovich number is shown as a function of the wave number for different values of the frontal Rayleigh number. We note that positive $R$ corresponds to ascending fronts, negative $R$ to descending fronts. The stable regions are below the corresponding curves, the unstable regions above.

In Fig. 2 the critical values of the Rayleigh number are shown as functions of wave number for different values of the Zeldovich number. The unstable regions correspond to large (in absolute value) Rayleigh numbers. We note that the instability is possible not only for ascending fronts $(R>0)$, but also for the descending fronts $(R<0)$. We discuss this and other results in Sec. IV. 


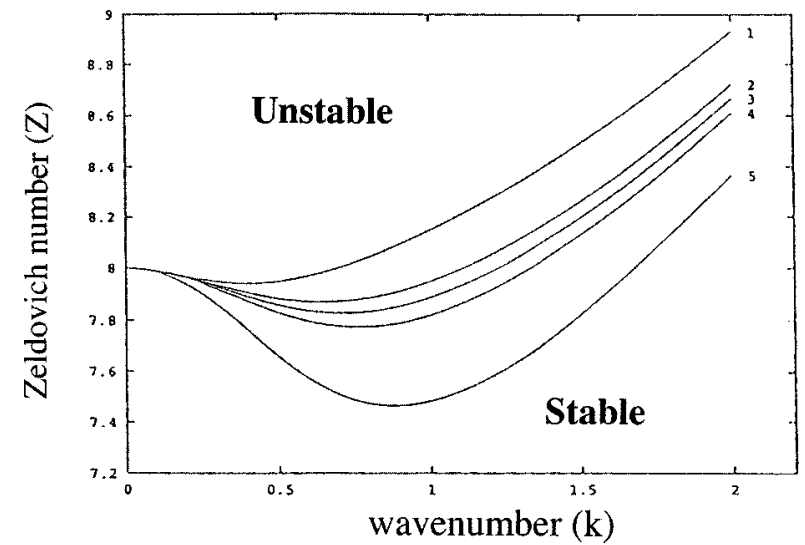

FIG. 1. Oscillatory instability boundary. $P=0.5,1 . R=-50,2 . R$ $=-10,3 . R=0,4 . R=10,5 . R=50$.

Figures 3 and 4 show the level lines of the stream function for ascending and descending fronts for the values of parameters that correspond to the cellular (convective) stability boundary and Figs. 5 and 6 to the values of parameters on the oscillatory (thermal) stability boundary.

\section{EXPERIMENTAL}

We were unable to study descending liquid/liquid polymerization fronts because double-diffusive convection interfered. ${ }^{29,31}$ Recall that the model assumes the density of the product only depends on the temperature and not upon the conversion. Addition polymerizations involve significant increases in density, as much as $20 \%$.

We performed experiments using acrylamide in dimethyl formamide (DMF) $(1: 1 \mathrm{w} / \mathrm{v})$ with benzoyl peroxide initiator. By adding ultrafine silica gel (CAB-O-SIL) we adjusted the initial viscosity. We studied the velocity of an ascending front as a function of the percentage CAB-O-SIL. Figure 7 shows that at low viscosity (and with convective motion that was clearly visible), the front was slower than at higher viscosity. At a critical value of the CAB-O-SIL, and hence the viscosity, the front velocity increased to a higher value at which it was then independent of viscosity.

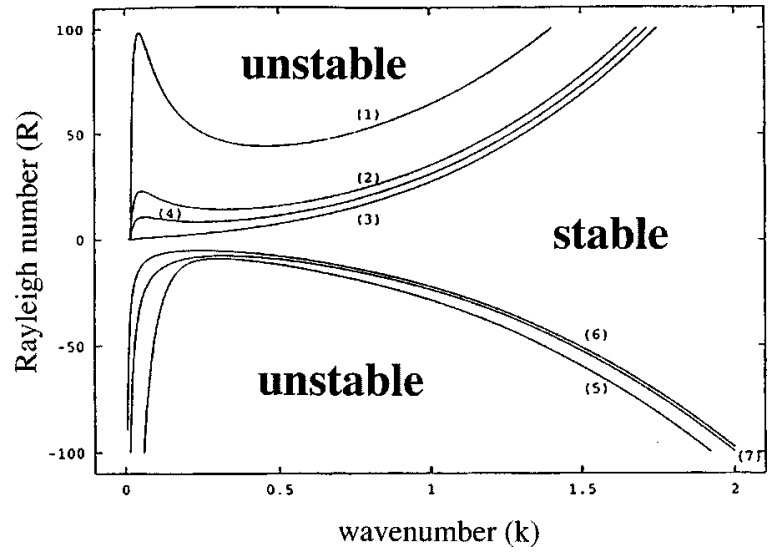

FIG. 2. Cellular instability boundary. $Z=160,1 . P=0.5,2 . P$ $=1.5$, 3. $P=10.5,4 . P=2.5, Z=16,5 . P=0.5,6 . P=2.5,7 . P=1.5$.

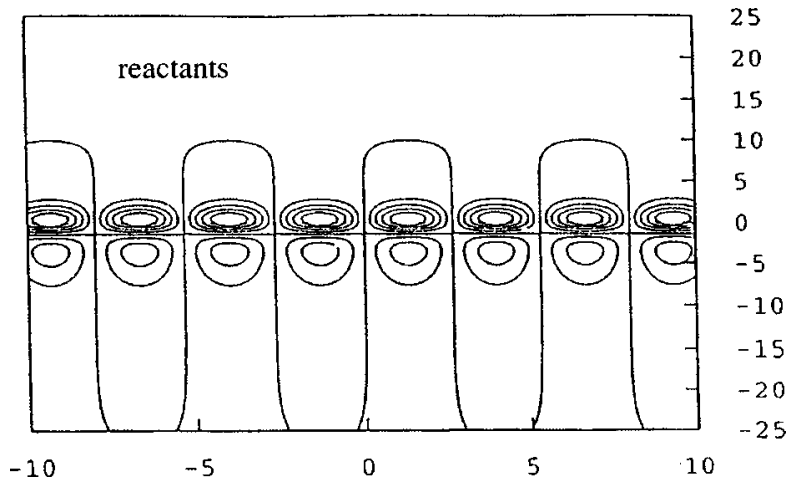

FIG. 3. Level lines of the stream function for an ascending front. Convective instability. $R=45, Z=8, P=0.5, k=1.785$.

Unfortunately, this system differed significantly from the model because the viscosity of the polymer solution was greatly different from that of the monomer solution. Moreover, as the polymer solution cooled, it gelled, creating a system that was liquid/viscous liquid/solid. We also observed another problem, as the initiator concentration was increased to increase the front velocity, the front became less stable, i.e., more convection was observed. We suspected this was caused by the decrease of molecular weight with the increase of initiator. Therefore we investigated another system.

To obtain a liquid-liquid system, benzyl acrylate in DMF was used. To maintain a low and constant molecular weight for all initiator concentrations, a chain transfer agent was used.

The chemicals, $N, N$-dimethylformamide (DMF) (Fisher), benzyl acrylate (BzA) (Monomer-Polymer and Dajac), 97\% benzoyl peroxide (BPO) (Aldrich), 98\% dodecanethiol (Aldrich), and amorphous fumed silica, CAB-OSIL (Cabot corporation) were used as received.

All experiments started with $10 \mathrm{~mL}$ of BzA dissolved in $10 \mathrm{~mL}$ of DMF. The pycnometery trials required a $60 \mathrm{~mL}$ batch using the same ratio. For the velocity trials, the initiator (BPO) was then added along with the corresponding amount of chain transfer agent. The relationship between the two chemicals was calculated based on information in Refs. 51 and 52. We used the following relationship, which was calculated to maintain a degree of polymerization of $5: 1$

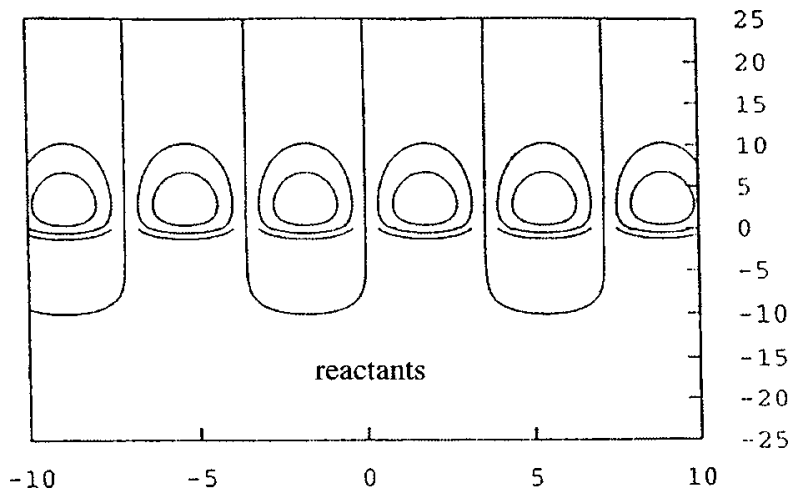

FIG. 4. Level lines of the stream function for a descending front. Convective instability. $R=-30, Z=8, P=0.5, k=0.88$. 


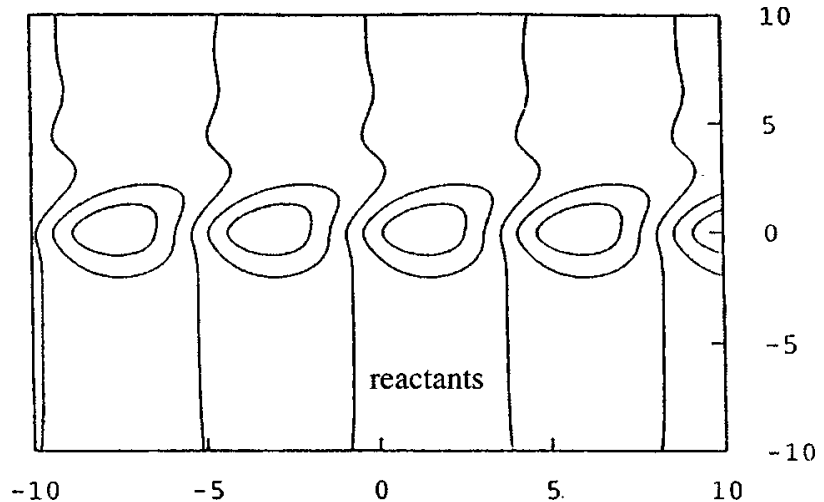

FIG. 5. Level lines of the stream function for a descending front. Thermal instability. $R=-50, Z=8, P=0.5, k=0.7$.

$=0.353 I+0.337 S$ where $I$ is in grams of benzoyl peroxide, and $S$ is the volume of dodecanethiol in microliters. The CAB-O-SIL was then added after being weighed out on an analytical balance.

\section{A. Viscosity measurements}

The viscometry runs as well as pycnometry runs did not contain initiator or chain transfer agent. Cannon-Fiske viscometers (sizes 25, 150, and 300) were used to measure the viscosity. The viscometers were used for the size of their capillaries and were not necessarily for their intended viscosity range. CAB-O-SIL tends to clog the capillaries and settle at lower concentrations, leading to low accuracy and reproducibility. All viscometry trials were repeated at least three times. The assays were run in a constant temperature water bath at $25.1{ }^{\circ} \mathrm{C}$ after charging the viscometer with solution and waiting for the system to equilibrate for $10 \mathrm{~min}$. The three efflux times were averaged and then multiplied by the viscometer constant provided by the manufacturer to obtain the kinematic viscosity. Selected CAB-O-SIL concentrations were also run in water baths heated to $50{ }^{\circ} \mathrm{C}$, and $72{ }^{\circ} \mathrm{C}$ and allowed to equilibrate for $30 \mathrm{~min}$ after having been charged.

The size 25 viscometer was used to determine the viscosity of the CAB-O-SIL-free solution. The size 300 viscometer was used for $4 \%$ to $5 \%$ CAB-O-SIL while the size 150 viscometer was used for $2.5 \%$ to $4 \%$ CAB-O-SIL. The viscometer constants provided by the manufacturer were used.

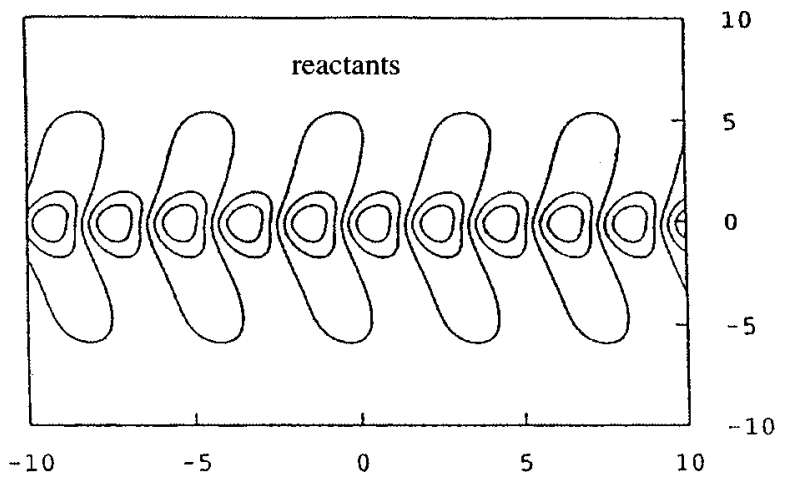

FIG. 6. Level lines of the stream function for an ascending front. Thermal instability. $R=10, Z=8, P=4.5, k=1.55$.
acrylamide/DMF

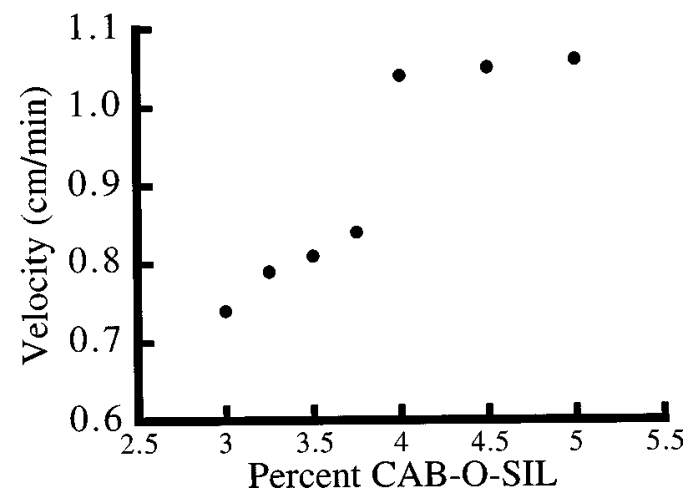

FIG. 7. The velocity of an ascending acrylamide polymerization front in $\operatorname{DMF}(1: 1 \mathrm{w} / \mathrm{v})$ as a function of the amount of added CAB-O-SIL. Benzoyl peroxide was the initiator.

The viscosity as a function of percentage CAB-O-SIL is shown in Fig. 8.

A $50 \mathrm{~mL}$ pycnometer was used to measure the thermal expansion coefficient, as was described previously. ${ }^{41}$ It varied from 0.00090 to 0.0015 from 25 to $75{ }^{\circ} \mathrm{C}$.

\section{B. Temperature profiles}

An unsheathed thermocouple (Omega TMQSS-020g-6) was used to determine the temperature of the front as described previously. ${ }^{41} 10 \% \mathrm{BPO} \mathrm{w} / \mathrm{v}$ and $5 \% \mathrm{CAB}-\mathrm{O}-\mathrm{SIL} \mathrm{w} / \mathrm{v}$ were used (Fig. 9).

\section{Standard procedure}

Bubble production (from the initiator decomposition) makes determination of the stability difficult because they affect both velocity and fluid motion. Therefore the type of tube is important. We used tubes $16 \times 125 \mathrm{~mm}$ (VWR No. 72690-022) on which a plastic cap (VWR No. 60826-290) could be securely screwed. In addition, the tube was sealed with epoxy and teflon tape. Care must be exercised because a few atmospheres of pressure can buildup during the reaction, and so all reactions should be performed behind a shield. (Out of 100 experiments, one tube exploded.) Our experiments were performed in an evacuated side arm flask, which

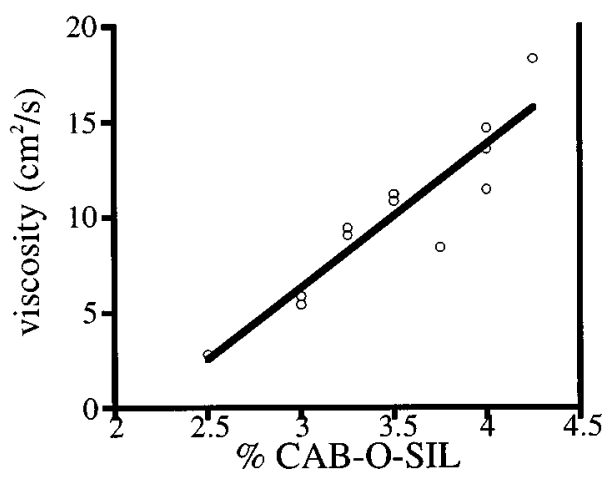

FIG. 8. The viscosity of a 1:1 benzyl acrylate/DMF solution as a function of percentage CAB-O-SIL at $25.1{ }^{\circ} \mathrm{C}$. 


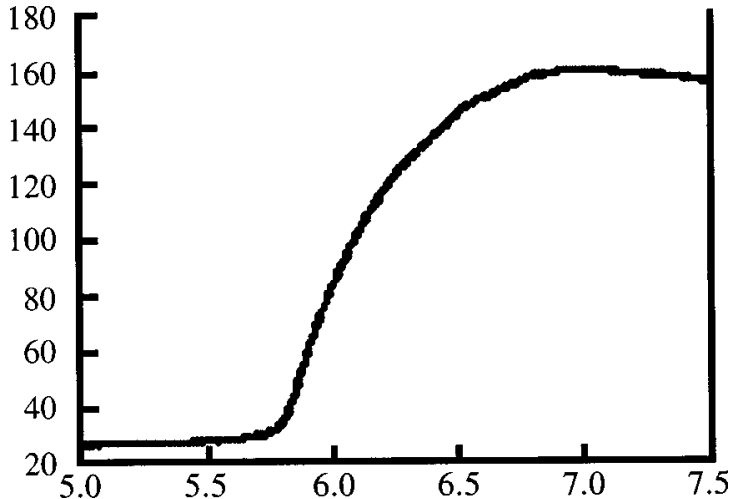

FIG. 9. The front temperature profile for a convection-free benzyl acrylate/ DMF front with $5 \%$ CAB-O-SIL and $10 \%$ BPO.

was behind a safety shield. Fronts were monitored remotely using a video camera. After the batch solution was made up but before the addition of the CAB-O-SIL, the solution was degassed under low pressure provided by a vacuum pump for 10 min. The CAB-O-SIL was then added. A layer of sand was placed on the bottom the test tube to provide infinite viscosity at the start of the reaction and to produce an initial level front. About $2 \mathrm{~mL}$ of solution was placed in the test tube, which was then centrifuged and degassed again for 15 min. The test tube was then filled with the reaction solution and degassed again for $45 \mathrm{~min}$ to $1 \mathrm{~h}$ after which the top of the tube was sealed with a cork that had silicon glue around the edges and on the top. After the cap was tightly secured, a layer of silicon tape finished off the test tube sealing. The solution was then placed in a constant temperature water bath at $25.1^{\circ} \mathrm{C}$ for about $10 \mathrm{~min}$ to equilibrate the test tube. The test tube was subsequently dried, and the front initiated with a modified soldering iron. The iron had the sharp tip removed to increase the surface area of the end. After the front was initiated, the test tube was placed in a filter flask with a rubber stopper placed on the top, which was then evacuated to reduce heat loss.

\section{Determining stability}

An unstable ascending liquid-liquid front can be determined by either front symmetry or velocity. In the presence of convection, the fronts have a slower velocity than a convection-free front. Determinations of convection free velocity and of the minimum viscosity necessary for a stable front were done from the plot of ascending velocity as a function of the initial viscosity. The convection-free velocity was estimated by taking the highest reproducible velocity over a wide range of viscosities. The viscosity range for the stability boundary was determined by taking the highest unstable point as the maximum viscosity and taking the lowest stable point as the minimum viscosity.

The ascending front velocity is very sensitive to the viscosity (Fig. 10). Below the critical viscosity the convection quenches the front. It is more sensitive than the acrylamide/ DMF system (Fig. 7), which makes sense because the product in that system was much more viscous than the reactant solution.

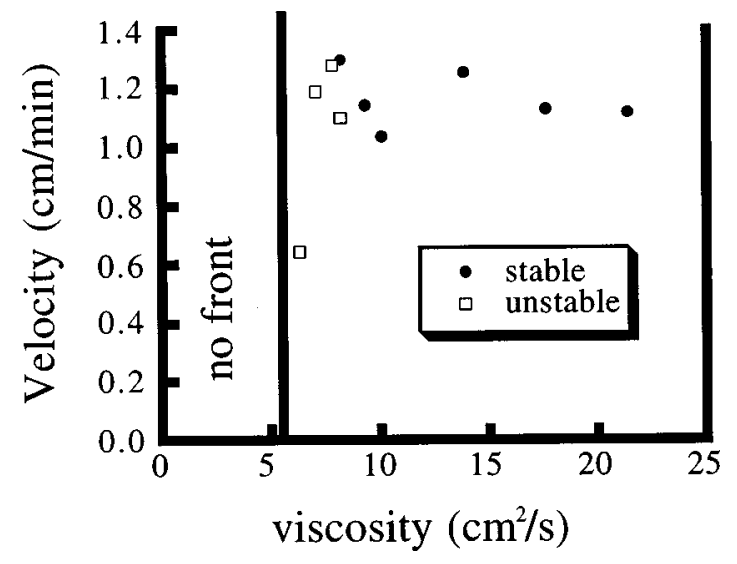

FIG. 10. The ascending front velocity as a function of the initial viscosity benzyl acrylate/DMF solution for $10 \% \mathrm{BPO}$.

The modes of convection than can arise in ascending fronts are shown in Fig. 11. The antisymmetric mode occurs near the stability boundary and the axisymmetric one farther from the stability boundary, exactly as was observed with liquid/solid fronts. ${ }^{41}$

Spin modes were not observed in descending fronts with solid product with a monoacrylate monomer at room temperature and higher front temperatures. ${ }^{6,45,53}$ Nonetheless, spin modes were observed for ascending fronts with $12 \%$ BPO and 3.5\% CAB-O-SIL. In solid product fronts, a spiral pattern is left in the product. In liquid product fronts, however, the pattern disappears after a short time. Figure 12 presents images of ascending and descending fronts near the stability boundary. Weak convection was observed in both cases but the ascending does not remain planar but appears
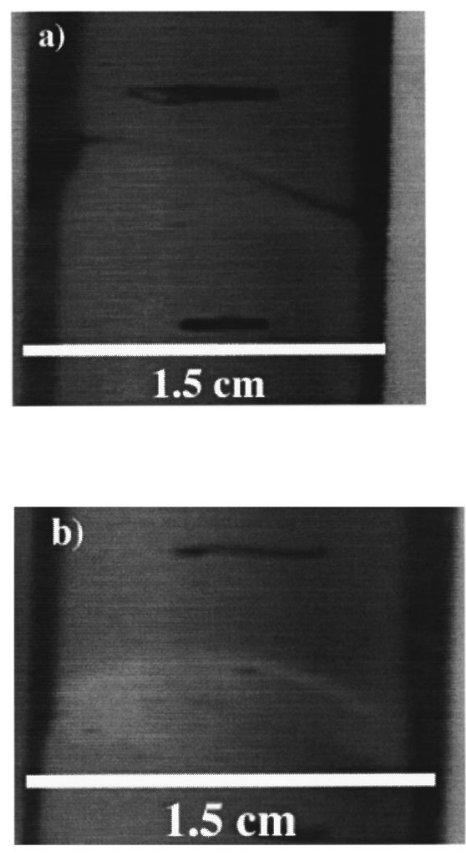

FIG. 11. (a) An antisymmetric ascending front, near the stability boundary. (b) Axisymmetric front, farther from the stability boundary. 

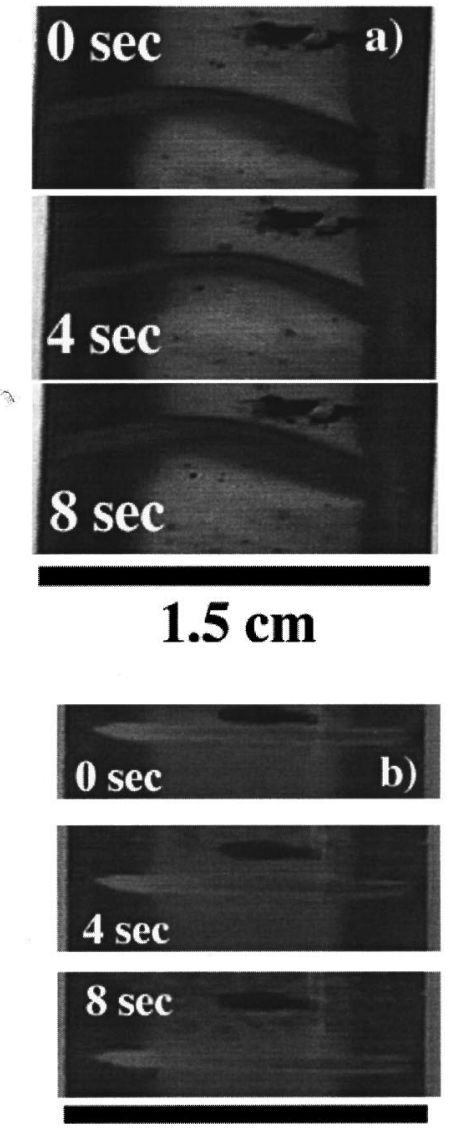

FIG. 12. (a) Spin mode in ascending front of benzyl acrylate polymerization in DMF; (b) planar descending front without spin mode. Both runs were with $12 \% \mathrm{BPO}$ and $3.5 \%$ CAB-O-SIL.

to wobble as the hot spot of the spin mode propagates around the front.

Using the energy of activation for methacrylic acid (such values are very similar for monoacrylates) and for benzoyl peroxide, the Zeldovich number can be calculated, as done in Refs. 45 and 53 to be about 6 , which is well below the critical value for spin modes. However, with a liquid product, a planar ascending front is less stable with respect to spin modes at low viscosity (see Fig. 1).

In Fig. 13 we compare the stability boundary calculated

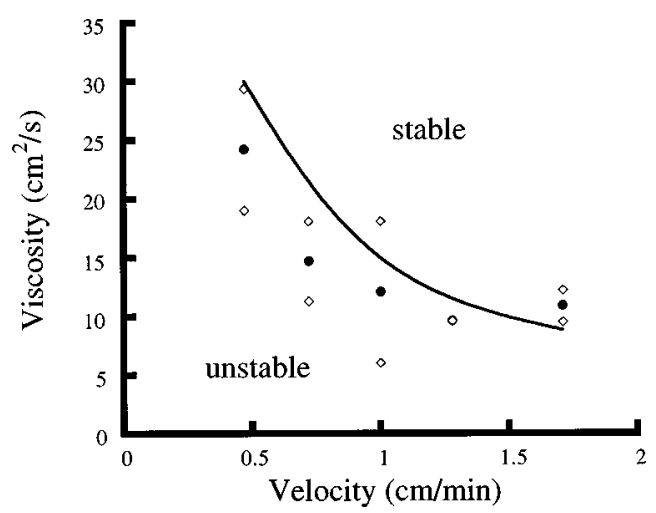

FIG. 13. Comparison of the experimental and theoretical stability boundary for an ascending front. theoretically to our experimentally determined one. The theoretical stability boundary is found using the results of Sec. II. From the expression for the frontal Rayleigh number we have

$$
\nu=\frac{g \beta q \kappa^{2}}{c^{3} R(k)},
$$

where $R(k)$ is the function that determines the stability boundary (see Fig. 2). For not very small values of $k$ and positive $R$ it can be approximated by the function $R(k)$ $=a k^{2}+b$ with good precision. Here $a$ is independent of other parameters and equals $28, b$ depends on $Z$ and $P$. It equals approximately 6.6 for $Z=160$ and $P=10$ and decreases when $Z$ decreases or when $P$ increases. So for more realistic values of $Z$ between 5 and 8 the value of $b$ is less than that given above.

All experiments are performed in cylindrical tubes with the diameter $L=1.5 \mathrm{~cm}$. To put them in correspondence with the theory we neglect the influence of the walls on stability conditions and consider the experimental convective pattern as a part of the periodic structure in the infinite medium. The characteristic dimension of the pattern is $L$, and it is related to the wave number $k$ by the formula

$$
L=\frac{2 \pi}{k} \frac{\kappa}{c} .
$$

Using the approximate formula for $R(k)$ and expressing $k$ through $L$, we finally obtain the theoretical stability boundary on the velocity-viscosity plane

$$
\nu=\frac{g \beta q \kappa^{2}}{a(2 \pi \kappa / L)^{2} c+b c^{3}} .
$$

We compute it for the experimental values of parameters $q=140 \mathrm{~K}, \kappa=0.06 \mathrm{~cm}^{2} / \mathrm{min}^{41}$, and $\beta=10^{-3} \mathrm{~K}$ taken as an approximate value from the experimental measurements discussed above. We put $b=0$ to obtain the upper bound of the theoretical instability region. Even in this case the stability boundary is very close to the experimental one (Fig. 13). The fact that the theoretical stability region is less than the experimental one can be explained by the influence of the walls that make the front more stable. The last experimental point at high velocity may be not precise because of the difficulty in eliminating bubbles at the high initiator concentration.

\section{DISCUSSION}

In this work we study exothermic reaction fronts propagating in liquids. We compare the theoretical results with experimental results for polymerization fronts. We analyze an interaction between thermal phenomena and hydrodynamics for ascending and descending fronts.

For the upwards propagating fronts the exothermic chemical reaction heats the liquid reactants from below. So we can expect that under some conditions a convective motion of the liquid can occur. We find analytically the critical condition when it appears. This critical condition can be expressed in the form $R>R_{c r}(P, u, k)$, i.e., the frontal Rayleigh number should exceed a critical value which depends on the Prandtl number, on the front velocity, and on the wave num- 
ber. As mentioned in Sec. II, the frontal Rayleigh number is closely connected to the usual Rayleigh number. The theoretically measured stability boundary gives a good approximation of the experimental stability boundary. In the theory the front is less stable and it can be explained by the stabilizing influence of the walls in the experiments.

Convection decreases the speed of propagation of an ascending front with a liquid polymer. It was observed experimentally (Sec. III) and numerically. ${ }^{44}$

Convective structure in the case of ascending fronts consists of two vortices in the vertical direction. One of them crosses the front and another one is behind the front.

For descending fronts the exothermic reaction heats the reactants from above. So we can expect that convection cannot occur in this case. However, theoretical results show that it does occur if the absolute value of the Rayleigh number is sufficiently large. (We recall that the Rayleigh number is negative for descending fronts.) We can give the following physical explanation of this phenomenon. For the plane stable descending front the temperature at the reaction zone and behind it (i.e., for greater $z$ ) equals the adiabatic temperature. Suppose that there is a small fluctuation of temperature at the front, and it becomes greater than adiabatic. In this case the perturbed temperature is greater than the temperature above the front, which can lead to weak convection. This weak convective motion brings fresh reactants to the reaction zone and the heat release because of the reaction increases the temperature even more. So we have a selfaccelerating process that can lead to the instability. This type of instability is not yet found experimentally.

The convective instability of a descending front appears as a result of interaction of the chemical reaction and hydrodynamics. It does not occur if the product of the reaction is solid. $^{42}$

The convective instability appears for large values of the frontal Rayleigh number. The results discussed above are obtained for the values of the Zeldovich number less than the critical value for the thermal instability. If, inversely, we take the value of the Rayleigh number less than the critical value for the convective instability and increase the Zeldovich number, then the thermal instability occurs. Conditions of the thermal instability depend on the Rayleigh number. If $Z_{c r}(R)$ is the critical value of the Zeldovich number, then $Z_{c r}(R)<Z_{c r}(0)$ for $R$ positive and $Z_{c r}(R)>Z_{c r}(0)$ for $R$ negative. It means that the natural convection makes ascending fronts less stable than without hydrodynamics and descending fronts more stable. It happens because the convective motion changes the temperature distribution around the front. This theoretical result is confirmed experimentally: the spinning modes for ascending fronts with a liquid polymer were observed for rather low values of the Zeldovich number (Sec. III).

It is interesting to note that the influence of the natural convection on the thermal instability is opposite in the case where the product of the reaction is solid. ${ }^{42}$ Not ascending but descending fronts are less stable in this case than those without hydrodynamics. These analytical results explain why the spinning modes were observed in the experiments on $\epsilon$-caprolactam polymerization. ${ }^{8}$ The value of the Zeldovich number in this case is small and according to the theory the front should be stable if it propagates in a solid phase.

\section{v. CONCLUSIONS}

In this work we study stability of reaction fronts in liquids and compare theoretical results with experiments on frontal polymerization. We introduce the frontal Rayleigh number and show that if it exceeds the critical value, then the plane ascending front loses its stability, and convective motion of the liquid around the front can appear. The theoretically found stability boundary is compared with the experimental one. They are in a good agreement. The stability conditions depend on the speed of the convection-free front. If the speed is greater, the front is more stable.

Stability analysis shows that for descending fronts convective instability can also occur. The result is surprising because in this case the exothermic chemical reaction heats the cold reactant from above. It is a new type of instability that appears as a result of interaction of chemistry and hydrodynamics. It is not yet found experimentally and it will require additional theoretical and experimental investigations.

We show that hydrodynamics influences conditions of the thermal instability. The front propagating upwards becomes less stable than without hydrodynamics, the front propagating downwards more stable. This theoretical result is in agreement with the experiments where the spinning modes for ascending fronts were observed for low Zeldovich numbers but not for the same conditions with a descending front.

\section{ACKNOWLEDGMENTS}

This work was supported by the Air Force Office of Scientific Research and from an NSF REU Grant (DMR9531507).

${ }^{1}$ N. M. Chechilo, R. Ya. Khvilivitsky, and N. S. Enikolopyan, Doklady Physical Chemistry 204, 512 (1972).

${ }^{2}$ N. M. Chechilo and N. S. Enikolopyan, Doklady Physical Chemistry 214, 174 (1974).

${ }^{3}$ N. M. Chechilo and N. S. Enikolopyan, Doklady Physical Chemistry 221, 391 (1975).

${ }^{4}$ N. M. Chechilo and N. S. Enikolopyan, Doklady Physical Chemistry 230, 840 (1976).

${ }^{5}$ S. P. Davtyan, P. V. Zhirkov, and S. A. Vol'fson, Russ. Chem. Rev. 53, 150 (1984).

${ }^{6}$ J. A. Pojman, V. M. Ilyashenko, and A. M. Khan, J. Chem. Soc., Faraday Trans. 92, 2825 (1996).

${ }^{7}$ Vit. Volpert and Vl. Volpert, European Journal of Applied Mathematics 5, 201 (1994).

${ }^{8}$ V. P. Begishev, V. A. Volpert, S. P. Davtyan, and A. Y. Malkin, Doklady Physical Chemistry 29, 1057 (1984).

${ }^{9}$ J. A. Pojman, R. Craven, A. Khan, and W. West, J. Phys. Chem. 96, 7466 (1992).

${ }^{10}$ V. A. Volpert, V. A. Volpert, S. Solovjov, and J. Pojman, European Journal of Applied Mathematics 7, 303 (1996).

${ }^{11}$ A. G. Merzhanov, A. K. Filonenko, and I. P. Borovinskaya, Dokl. Akad. Nauk SSSR 208, 892 (1973).

${ }^{12}$ A. P. Aldushin, T. M. Martemyanova, A. G. Merzhanov, B. I. Khaikin, and K. G. Shkadinskii, Combust., Explos. Shock Waves 9, 531 (1973).

${ }^{13}$ A. Bayliss and B. J. Matkowsky, SIAM (Soc. Ind. Appl. Math.) J. Appl. Math. 50, 437 (1990).

${ }^{14}$ T. P. Ivleva, A. G. Merzhanov, and K. G. Shkadinskii, Combust., Explos. Shock Waves 16, 133 (1980).

${ }^{15}$ S. B. Scherbak, Combust., Explos. Shock Waves 19, 542 (1983). 
${ }^{16}$ K. G. shkadinskii, B. I. Haikin, and A. G. Merzhanov, Combust., Explos. Shock Waves 7, 15 (1971).

${ }^{17}$ V. A. Volpert, V. A. Volpert, S. P. Davtyan, I. N. Megrabova, and N. F. Surkov, SIAM (Soc. Ind. Appl. Math.) J. Appl. Math. 52, 368 (1992).

${ }^{18}$ A. P. Aldushin and S. G. Kasparyan, Dokl. Akad. Nauk SSSR 244, 67 (1979) (in Russian).

${ }^{19}$ B. J. Matkowsky and G. I. Sivashinsky, SIAM (Soc. Ind. Appl. Math.) J. Appl. Math. 35, 465 (1978)

${ }^{20}$ B. V. Novozhilov, Pure Appl. Chem. 65, 309 (1995).

${ }^{21}$ A. I. Volpert, V. A. Volpert, and V. A. Volpert, Traveling Wave Solutions of Parabolic Systems, (American Mathematical Society, Providence, 1994), Vol. 140

${ }^{22}$ G. I. Barenblatt, Ya. B. Zeldovich and A. G. Istratov, Zh Prikl Mekh. Tekh. Fiz. 21 (1962) (in Russian).

${ }^{23}$ A. Bayliss and B. J. Matkowsky, SIAM (Soc. Ind. Appl. Math.) J. Appl. Math. 52, 396 (1992).

${ }^{24}$ P. Clavin, Prog. Energy Combust. Sci. 11, 1 (1985).

${ }^{25}$ Ya. B. Zeldovich, G. I. Barenblatt, V. B. Librovich, and G. M. Makhviladze, The Mathematical Theory of Combustion and Explosions (Consultants Bureau, New York, 1985).

${ }^{26}$ B. J. Matkowsky and G. I. Sivashinsky, SIAM (Soc. Ind. Appl. Math.) J. Appl. Math. 37, 669 (1979).

${ }^{27}$ B. F. Edwards, J. W. Wilder, and K. Showalter, Phys. Rev. A 43, 749 (1991)

${ }^{28}$ J. Masere, D. A. Vasquez, B. F. Edwards, J. W. Wilder, and K. Showalter, J. Phys. Chem. 98, 6505 (1994).

${ }^{29}$ J. A. Pojman and I. R. Epstein, J. Phys. Chem. 94, 4966 (1990).

${ }^{30}$ J. A. Pojman, I. R. Epstein, T. J. McManus, and K. Showalter, J. Phys. Chem. 95, 1299 (1991).

${ }^{31}$ J. A. Pojman, I. P. Nagy, and I. R. Epstein, J. Phys. Chem. 95, 1306 (1991).

${ }^{32}$ D. A. Vasquez, J. W. Wilder, and B. F. Edwards, J. Chem. Phys. 98, 2138 (1993).

${ }^{33}$ D. A. Vasquez, B. F. Edwards, and J. W. Wilder, Phys. Rev. A 43, 6694 (1991).

${ }^{34}$ D. A. Vasquez, J. M. Littley, J. W. Wilder, and B. F. Edwards, Phys. Rev. E 50, 280 (1994).
${ }^{35}$ D. A. Vasquez, J. M. Littley, J. W. Wilder, and B. F. Edwards, Phys. Rev. 50, 280 (1994).

${ }^{36}$ J. W. Wilder, B. F. Edwards, D. A. Vasquez, and G. I. Sivashinsky, Physica D 73, 217 (1994).

${ }^{37}$ D. Gutkowicz-Krusin and J. Ross, J. Chem. Phys. 72, 3577 (1980).

${ }^{38}$ D. Gutkowicz-Krusin and J. Ross, J. Chem. Phys. 72, 3588 (1980).

${ }^{39}$ J. H. Viljoen, J. E. Gatica, and V. Hlavacek, Chem. Eng. Sci. 45, 503 (1990).

${ }^{40}$ F. A. Williams. Combustion Theory, 2nd ed. (Benjamin/Cumming, Menlo Park, 1985).

${ }^{41}$ G. Bowden, M. Garbey, V. Ilyashenko, J. Pojman, S. Solovyov, A. Taik, and V. Volpert, J. Phys. Chem. B 101, 678 (1997).

${ }^{42}$ M. Garbey, A. Taik, and V. Volpert, Q. Appl. Math. 54, 225 (1996).

${ }^{43}$ M. Garbey, A. Taik, and V. Volpert, Q. Appl. Math. 56, (1998).

${ }^{44}$ M. Garbey, A. Taik, and V. Volpert, in Numerical Methods for Fluid Dynamics V, edited by K. W. Morton and M. J. Baines (Clarendon, Oxford, 1995), pp. 415-421.

${ }^{45}$ J. A. Pojman, V. M. Ilyashenko, and A. M. Khan, Physica D 84, 260 (1995).

${ }^{46}$ V. A. Volpert, I. N. Megrabova, S. P. Davtyan, and V. P. Begishev, Combust., Explos. Shock Waves 21, 441 (1985).

${ }^{47}$ Vit. Volpert, Vl. Volpert, M. Garbey, and J. Pojman, in Gas Phase Chemical Reaction Systems, edited by J. Wolfrum, H.-R. Volpp, R. Rannacher, and J. Warnatz (Springer Series in Chemical Physics, Vol. 61, Springer, 1996), 309-317.

${ }^{48}$ Ya. B. Zeldovich and D. A. Frank-Kamenetsky, Zh. Fiz. Khim. 12, 100 (1938) (in Russian).

${ }^{49}$ B. V. Novozhilov, Proceedings of the Academy Sciences of the USSR, Physical Chemistry Section 141, 836 (1961).

${ }^{50} \mathrm{~W}$. Eckhaus, Asymptotic Analysis of Singular Perturbations (NorthHolland, Amsterdam, 1979.)

${ }^{51}$ Polymer Handbook, edited by J. Brandrup and E. H. Immergut (Wiley, New York, 1989)

${ }^{52}$ G. Odian, Principles of Polymerization (Wiley, New York, 1991).

${ }^{53}$ V. M. Ilyashenko and J. A. Pojman, Chaos 8, 285-289 (1998). 
Chaos is copyrighted by AIP Publishing LLC (AIP). Reuse of AIP content is subject to the terms at: http://scitation.aip.org/termsconditions. For more information, see http://publishing.aip.org/authors/rights-and-permissions. 